\title{
Pitfall of Positron Emission Tomography-Computed Tomography in assessing Metastasis in a Recurrent Head and Neck Cancer
}

\author{
${ }^{1}$ Poonam Joshi, ${ }^{2}$ Pankaj Chaturvedi, ${ }^{3}$ Jai Prakash Agarwal, ${ }^{4}$ Shubhada Kane
}

\begin{abstract}
Advanced and recurrent head and neck cancers often present with distant metastasis. The most common sites include lung, liver and bones. The propensity for metastasis depends upon the site of the primary disease as well. Cancers of hypopharynx and nasopharynx both have high propensity for distant metastasis. Positron emission tomography-computed tomography is done in many of these situations to assess distant metastasis, especially in locally advanced and recurrent cases.

The positive predictive value of PET-CT in predicting distant metastasis in recurrent head and neck cancers is $60 \%$. This low positive predictive value is attributed to inflammation, infections, and post RT changes. The present case report highlights that PET positive lesions should be interpreted with caution as they can be false positive and can be mistaken for metastasis and, hence, a potentially curative patient can be treated as one with palliative intent.
\end{abstract}

Keywords: PET-CT, Distant metastasis, False positive, Head and neck cancer.

How to cite this article: Joshi P, Chaturvedi P, Agarwal JP, KaneS. Pitfall of Positron Emission Tomography-Computed Tomography in assessing Metastasis in a Recurrent Head and Neck Cancer. Int J Head Neck Surg 2015;6(1):14-16.

Source of support: Nil

Conflict of interest: None

\section{INTRODUCTION}

Positron emission tomography-computed tomography (CT) scan has become the main imaging modality for detection of distant metastasis in locally advanced cancers, recurrent tumors and postradiotherapy follow-up of head and neck cancers. PET-CT is particularly useful in planning radical surgery in advanced and recurrent head and neck cancers. Head and neck cancers have distant metastasis rate of 6 to $10 \% .{ }^{1}$ However, the incidence of

\footnotetext{
${ }^{1}$ Adhoc Lecturer, ${ }^{2-4}$ Professor

${ }^{1-3}$ Department of Head and Neck Surgery, Tata Memorial Hospital, Navi Mumbai, Maharashtra, India

${ }^{4}$ Department of Pathology, Tata Memorial Hospital, Navi Mumbai Maharashtra, India

Corresponding Author: Poonam Joshi, Adhoc Lecturer Department of Head and Neck Surgery, Tata Memorial Hospital, Navi Mumbai, Maharashtra, India, Phone: 9769143523, e-mail: drpunamjoshi@rediffmail.com
}

distant metastasis in recurrent head and neck cancers was $23 \%$ with $20 \%$ unsuspected prior to PET-CT. ${ }^{2}$

But, the incidence of coexistent inflammatory and infectious lesions, like tuberculosis, is high in developing countries. ${ }^{3,4}$ The tubercular lesions accumulate ${ }^{18} \mathrm{~F}$-FDG due to active glucose metabolism and thus cause PET scans to be interpreted as false-positive for malignancy. ${ }^{5}$

\section{CASE REPORT}

A 76-year-old female presented to head and neck oncology clinic with a history of recurrent lesion in left buccal mucosa. She was a case of carcinoma of left buccal mucosa for which she underwent wide excision outside in 2007. She developed lesion in left buccal mucosa again in 2011. She was operated outside with wide excision of buccal mucosa with pectoralis major myocutaneous (PMMC) flap in 2011. Histopathology was suggestive of poorly differentiated squamous cell carcinoma. After 2 years, she again developed lesion in left buccal mucosa and this time she sought opinion at our oncology clinic.

Positron emission tomography-computed tomography scan was done for assessment of distant metastasis status. PET scan was suggestive of FDG avid enhancing soft tissue thickening in the left GB sulcus involving the left RMT, eroding the left mandibular ramus, measuring $2.1 \times 1.0 \mathrm{~cm}$ with max SUV of 6.6. FDG avid enhancing nodes were also noted in the left supraclavicular nodes $(1 \mathrm{~cm}$, max SUV 4.5), multiple gastrohepatic nodes, periportal nodes and multiple small para-aortic and para caval nodes. Multiple peripherally enhancing hypodense lesions were noted in the spleen parenchyma (largest at the anterior end, measures $2.1 \mathrm{~cm}$, max SUV 4.5). All these were reported as metastatic.

Biopsy was taken from the buccal mucosa (Fig. 1) and was suggestive of squamous carcinoma. Fine needle aspiration cytology (FNAC) of the supraclavicular node (SCLN) was reactive (Fig. 2) and FNAC from the spleen (Figs 3 and 4) was suggestive of mycobacterial Koch's infection. There was discordance between FNAC of SCLN (negative), spleen (negative) and FDG PET CT (positive) findings, with PET-CT findings being false positive. Patient was planned for antitubercular treatment followed by radical treatment. 


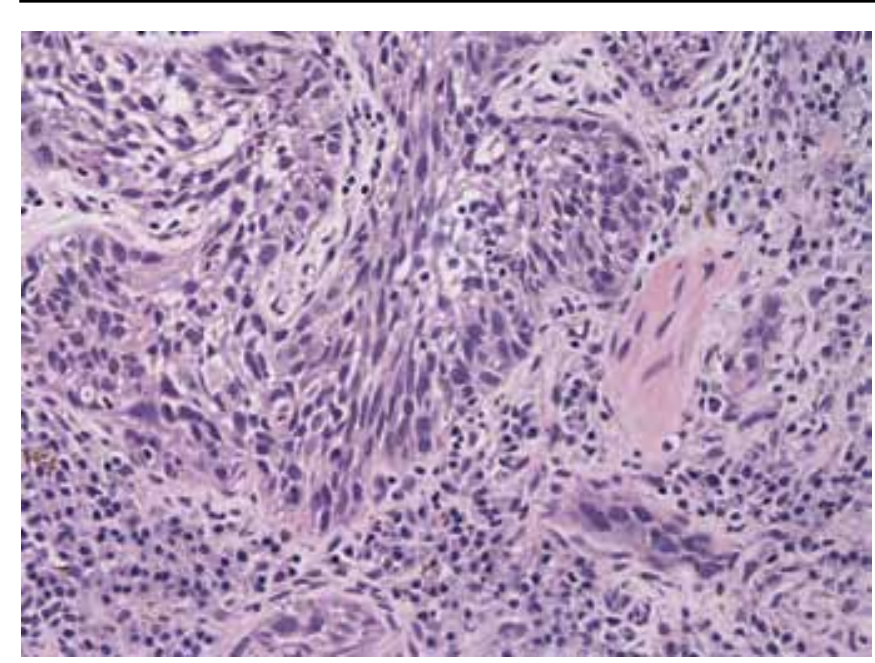

Fig. 1: Slide of buccal mucosa biopsy shows invasive squamous cell carcinoma (H\&E: 40x)

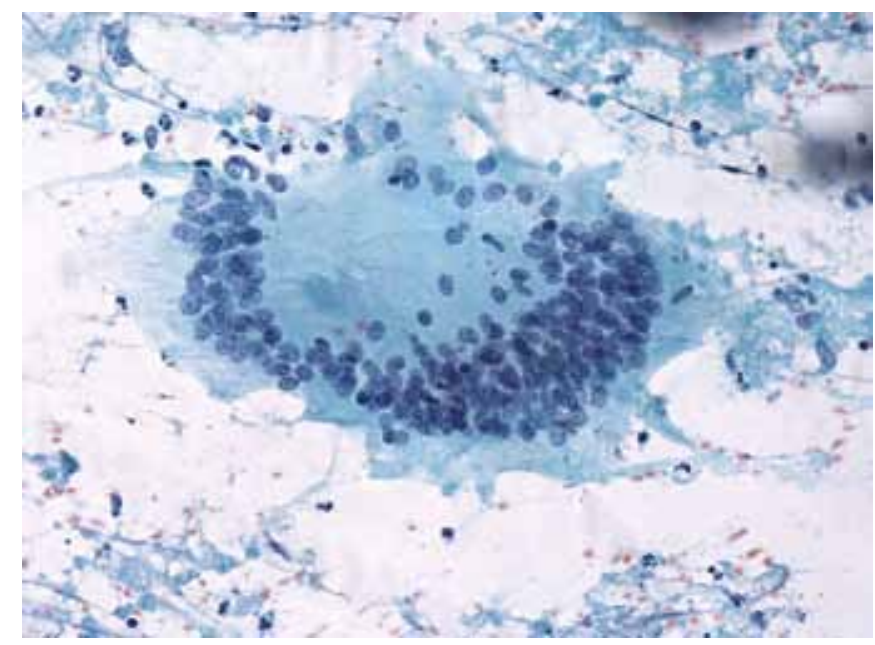

Fig. 3: Slide of spleen FNAC showing foreign body giant cell (H\&E)

\section{DISCUSSION}

Distant metastasis is a common feature in advanced and recurrent head and neck cancers. Coexistence of inflammatory lesions with cancers of head and neck on PET-CT scan often mimics metastasis.

There are several studies evaluating the false positive results in head and neck cancers. ${ }^{1,6,7}$ False positivity is seen with PET-CT scan in cases of inflammatory lesions, infections, postsurgery and postradiotherapy setting. The FDG avidity of spleen and neck nodes in a proven carcinoma of head and neck can suggest distant metastasis. But, this needs to be taken with caution as this can be inflammatory and be mistaken for metastasis and a resectable and potentially curable patient can be planned for palliative therapy, particularly in developing countries where the incidence of tubercular infection is high.

Spleen is an unusual site for metastasis for head and neck cancer. Moreover, in absence of involvement of other common sites, should be evaluated and tissue diagnosis should be done. Isolated splenic tuberculosis is also rarely

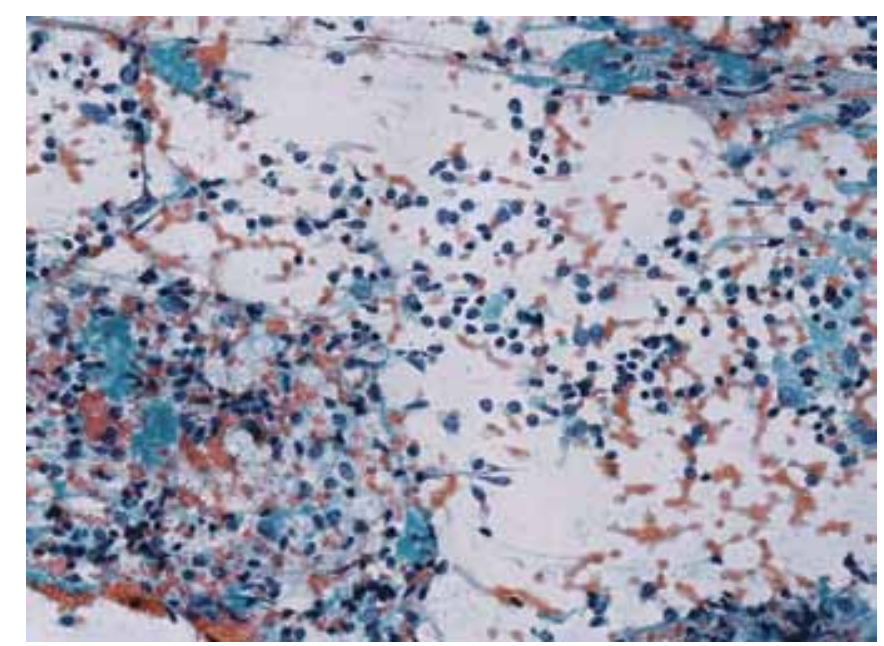

Fig. 2: Slide of supraclavicular LN FNAC showing reactive lymphoid aspirate (H\&E: 40x)

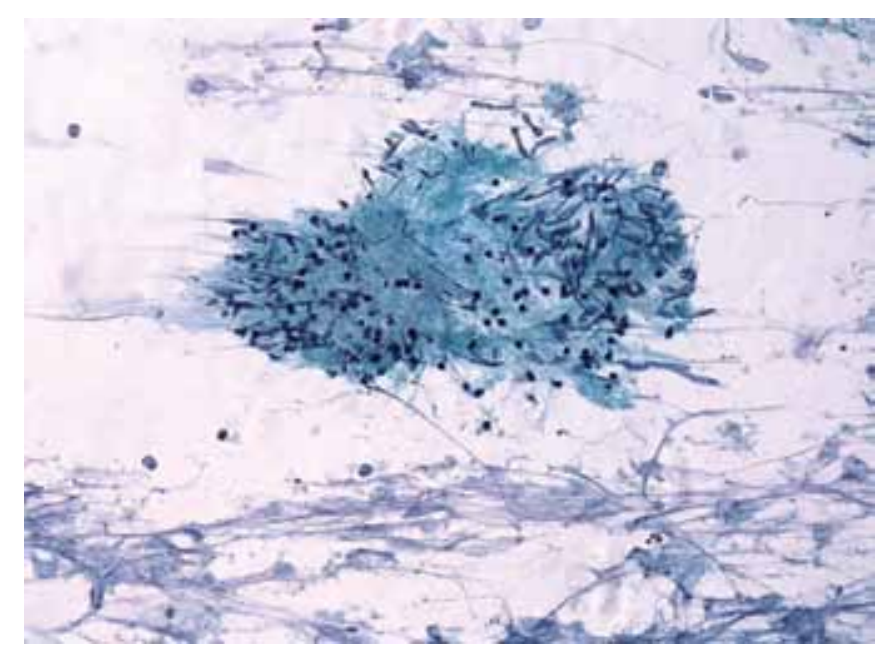

Fig. 4: Slide of spleen FNAC showing granuloma formation (H\&E)

reported. ${ }^{8}$ Splenic tuberculosis usually occurs as a part of disseminated disease. CT scan in these situations is suggestive of multiple small hypodense splenic nodules. But, histological and microbiological confirmation is usually needed to confirm the tuberculous etiology.

The present case highlights the pitfalls of PET-CT scan in evaluation of head and neck cancers. The incidence of coexistent tuberculosis is common in our country. PET also shows uptake in infectious and inflammatory lesions. One should be aware of this false positivity of PET-CT scan report. Results of PET-CT scan should be interpreted cautiously along with clinical judgment and proper evaluation before changing decision particularly in a potentially curable patient. Such lesions should undergo histopathological examination before changing treatment plan and may require follow-up.

\section{REFERENCES}

1. Goerres GW, Schmid DT, Gratz KW, Von Schulthess GK, Eyrich GK. Impact of whole body positron emission tomography on initial staging and therapy in patients with 
squamous cell carcinoma of the oral cavity. Oral Oncology 2003;39(6):547-551.

2. Gourin CG, Watts T, Williams HT, Patel VS, Bilodeau PA, Coleman TA. Identification of distant metastases with PET$\mathrm{CT}$ in patients with suspected recurrent head and neck cancer. Laryngoscope 2009;119(4):703-706.

3. Cegielski JP, Chin DP, Espinal MA, Frieden TR, Rodriquez Cruz R, Talbot EA, et al. The global tuberculosis situation: progress and problems in the 20th century, prospects for the 21st century. Infect Dis Clin North Am 2002;16(1): 1-58.

4. World Health Organization. Fact sheet no. 104 Tuberculosis. Available at: www.who.int/mediacentre/factsheets/fs104. WHO Website. Revised on: March 2007. Accessed on: May 21, 2008.
5. Goo JM, Im JG, Do KH, Yeo JS, Seo JB, Kim HY, et al. Pulmonary tuberculoma evaluated by means of FDG PET: findings in 10 cases. Radiology 2000;216(1):117-121.

6. SchwartzDL, RajendranJ, YuehB, Coltrera M, Anzai Y,KrohnK, et al. Staging of head and neck squamous cell cancer with extended-field FDG-PET. Arch Otolaryngol Head Neck Surg 2003 Nov;129(11):1173-1178.

7. Ng SH, Chan SC, Yen TC, Chang J TC, Liao CT, Ko SF, et al. Staging of untreated nasopharyngeal carcinoma with PET-CT: comparison with conventional imaging workup. Europ J Nucl Med Molec Imag 2009;36(1):12-22.

8. Ho PL, Chim CS, Yuen KY. Isolated splenic tuberculosis presenting with pyrexia of unknown origin. Scand J Infect Dis 2000;32(6):700-701. 